\title{
Therapie der akuten traumatischen Rückenmarkslähmung
}

\author{
Andreas Badke
}

\section{Zusammenfassung}

Die wesentlichen Ziele der Therapie der akuten traumatischen Querschnittlähmung sind die Vermeidung von Sekundärschäden und die Schaffung optimaler Bedingungen für eine Regeneration des Rückenmarks. Der Herstellung und Erhaltung einer stabilen Kreislaufsituation zur Vermeidung einer systemischen oder lokalen Hypoxie kommt dabei wesentliche Bedeutung zu. Die operative Therapie der Wirbelsäulenverletzung sollte eine möglichst frühe und vollständige Dekompression des Myelons herbeiführen. Gleichzeitig sollte eine Stabilisierung der Wirbelsäule zur Herstellung der Rehabilitationsfähigkeit durchgeführt werden. Die Gabe hoher Dosen Methylprednisolon kann eine sinnvolle Maßnahme sein, wird aber nicht mehr generell empfohlen.

\section{Therapy for Acute Traumatic Spinal Cord Injury}

The main goals of therapy for acute traumatic spinal cord injury are the avoidance of secondary damage and neurological deterioration as well as the maintenance of optimal conditions for the regeneration of the myelon. The avoidance of systemic and/or local hypoxia is essential. An operative therapy has to result in a sufficient decompression of the myelon as quickly as possible. Furthermore, the spine should be stabilised in order to facilitate the possibility of early rehabilitation. Administration of high-dose methylprednisolone can no longer be recommended as a general rule but can be considered for some selected patients.

\section{Einleitung}

Die Definition einer Querschnittlähmung umfasst spinale Funktionsausfälle als Folge einer Schädigung motorischer, sensibler und vegetativer Bahnen des Rückenmarks. Unterschieden werden komplette und inkomplette Lähmungsbilder. Bei einer kompletten Querschnittlähmung sind sämtliche Leitungsbahnen auf Höhe des Querschnittniveaus unterbrochen, sodass eine vollständige motorische Lähmung und ein ebenso vollständiger Ausfall der sensiblen Funktionen unterhalb des Lähmungsniveaus besteht. Bei inkompletten Querschnittlähmungen bestehen je nach Ausmaß der Traumatisierung des Rückenmarks unterschiedliche Mischbilder aus motorischen

OP-JOURNAL 2009; 25: 34-37

(c) Georg Thieme Verlag KG Stuttgart · New York DOI 10.1055/s-0029-1185495

und sensiblen sowie vegetativen Ausfallmustern [10].

Pathophysiologisch kann eine primäre von einer sekundären Zellschädigung unterschieden werden. Primär kommt es durch die Traumatisierung des Rückenmarks zu einer Zerreißung der Axone der langen Bahnen und/oder zu einer Zellschädigung mit Unterbrechung der elektrischen Leitungsfunktion der Axone. Sekundär kann eine Ausbreitung der Rückenmarksschädigung durch zusätzliche Entzündungsreaktionen sowie durch eine systemisch oder lokal bedingte Hypoxie erfolgen.

In den Industriestaaten wird von einer Inzidenz einer akuten traumatischen Rückenmarksläsion von 10-30 Fällen pro 1 Million Einwohner ausgegangen. Der Arbeitskreis „Querschnittlähmungen“ des Hauptverbands der gewerblichen Berufsgenossenschaften erfasst seit mehr als 30 Jahren alle Patienten, die in den deutschen Querschnittzentren aufgenommen werden. Die Auswertung dieser Zahlen ergibt, dass der Anteil der Tetraplegiker am Gesamtaufkommen frischer Querschnittlähmungen mit relativ geringen Schwankungen zwischen 35 und 40\% liegt. Männer sind mit $70 \%$ deutlich häufiger betroffen als Frauen. Verkehrsunfälle stellen nach wie vor die häufigste Ursache einer frischen Querschnittlähmung dar [3].

\section{Klinische Symptomatik}

Im Vordergrund der klinischen Symptome stehen die sensomotorischen Ausfälle unterhalb des Lähmungsniveaus sowie die klinischen Zeichen der Wirbelsäulenverletzung. Bei der akuten Querschnittlähmung treten jedoch zahlreiche weitere Störungen auf, die bei der Akuttherapie berücksichtigt werden müssen. Aufgrund der gestörten Verbindung zwischen dem sympathischen Nervensystem und dem Gehirn kommt es zu einer relativen parasympathischen Überaktivität. Hieraus ergibt sich eine Kreislaufregulationsstörung mit Hypotension und Neigung zur Bradykardie. Diese Regulationsstörungen sind bei Halsmarkschädigungen besonders ausgeprägt und können bei polytraumatisierten Patienten das Schockgeschehen zusätzlich negativ beeinflussen. Des Weiteren besteht eine Störung der Blasen- und Mastdarmfunktion. In der Phase des spinalen Schocks kommt es zu einer schlaffen Blasenlähmung.

Insbesondere bei zervikalen, aber auch bei hoch thorakalen Läsionen besteht eine ausgeprägte Einschränkung der Atemfunktion, bedingt durch Ausfall der thorakalen Atem- bzw. Atemhilfsmuskulatur. Bei zervikalen Läsionen zwischen C4 und C8 kommt es zu einer isolierten Zwerchfellatmung. Bei Läsionen oberhalb C4 kann auch die Zwerchfellfunktion ausfallen, sodass eine maschinelle Beatmung erforderlich wird. Aufgrund 


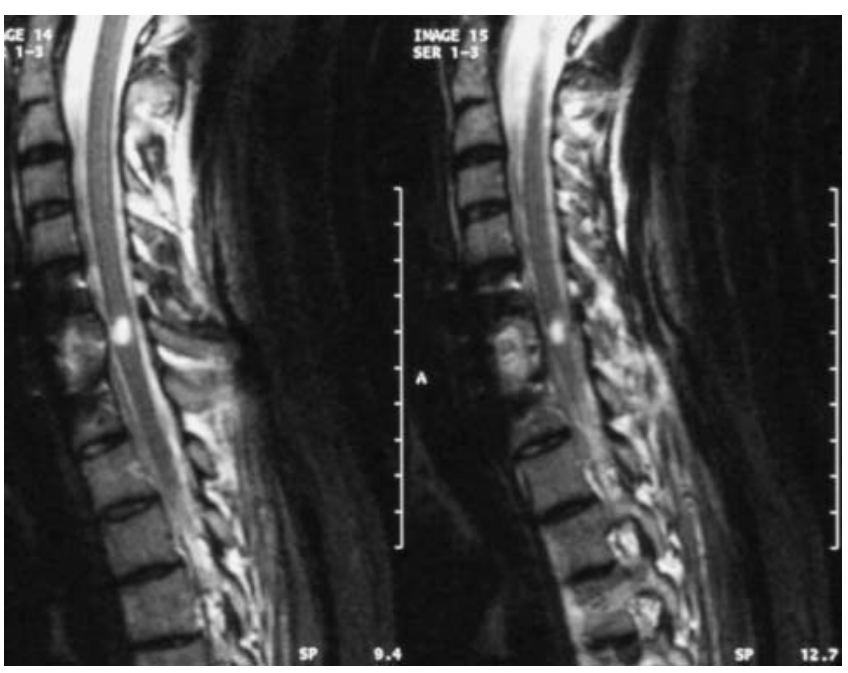

der vegetativen Dysregulation kommt es zusätzlich zu Temperaturregulationsstörungen, wobei in Abhängigkeit von der Lähmungshöhe sowohl hypo- als auch hypertherme Regulationsstörungen beobachtet werden können [10].

\section{Diagnostik}

Eine akute traumatische Läsion des Rückenmarks bedarf einer zielgerichteten, auf eine effiziente Therapiesteuerung ausgerichteten Diagnostik. Beim bewusstseinsklaren, kreislaufstabilen $\mathrm{Pa}$ tienten ist eine neurologische Untersuchung nach dem Untersuchungsprotokoll der American Spinal Injury Association (ASIA) durchzuführen, um das Lähmungsausmaß exakt zu dokumentieren. Im Rahmen der weiteren Diagnostik ist die Ursache der traumatischen Querschnittlähmung abzuklären. Besteht der Verdacht auf eine Wirbelsäulenverletzung, sind Röntgenaufnahmen der gesamten Wirbelsäule in 2 Ebenen anzufertigen. Gelingt die Darstellung des zervikothorakalen Übergangs nicht ausreichend, ist eine Erweiterung der Diagnostik mittels Comutertomografie unabdingbar. Bei polytraumatisierten Patienten ist in den letzten Jahren die Primärdiagnostik mittels Spiral-CT in den Vordergrund getreten, da diese eine lückenlose Dokumentation der gesamten Wirbelsäule im Schnittbildverfahren ermöglicht und somit einen raschen Überblick über etwaige Wirbelsäulenverletzungen gibt.

Sollte in den primär nativradiologisch durchgeführten Aufnahmen der Verdacht auf eine Wirbelfraktur bestehen oder diese nachgewiesen sein, ist die weitere computertomografische Abklädern (Abb. 1).
Abb. 1 MRT 2 Jahre nach operativer Behandlung einer Luxation C6/7 mit initial inkompletter Tetraplegie ASIA B und annähernd vollständiger Rückbildung der neurologischen Defizite trotz deutlich erkennbarem Rückenmarksschaden.

rung zur Planung eines etwaigen operativen Prozederes sinnvoll. Sind sowohl nativradiologisch als auch computertomografisch bei bestehender neurologischer Ausfallssymptomatik keine Wirbelsäulenverletzungen nachzuweisen, so ist in Abhängigkeit vom Lähmungsniveau eine Kernspintomografie anzuschließen. Diese ist sowohl in der Lage, den Rückenmarksschaden bildgebend darzustellen, als auch intraspinale Raumforderungen zum Beispiel durch Bandscheibenläsionen oder epidurale Einblutungen aufzudecken. Des Weiteren können durch die Kernspintomografie ligamentäre Verletzungen nachgewiesen werden, die insbesondere an der Halswirbelsäule auch ohne begleitende knöcherne Verletzungen auftreten können und eine umgehende Behandlung erfor-

\section{Therapie der akuten Querschnittlähmung}

Die Therapie der akuten Querschnittlähmung orientiert sich an 2 Zielen. Zum einen sollen Sekundärschäden am Rückenmark mit Verschlechterung der neurologischen Symptomatik vermieden werden. Zum anderen sollen die Bedingungen für eine Regeneration der Leitungsfunktion der betroffenen Nervenbahnen optimiert werden.

\section{Ziele der Therapie der akuten Querschnittlähmung}

1. Vermeidung von Sekundärschäden.

2. Herstellung optimaler Bedingungen für eine neurologische Regeneration.

Unter dem Schlagwort „Rehabilitation beginnt am Unfallort“" werden die bereits präklinisch durchzuführenden therapeutischen Maßnahmen bei einer akuten traumatischen Querschnittlähmung zusammengefasst. Bereits am Unfallort ist auf eine optimale Lagerung des Patienten (Vakuummatratze, Stiff Neck) zu achten, um Sekundärschäden am Rückenmark durch eine weitere Dislokation der Wirbelsäulenverletzung während des Transports zu vermeiden. Um die Sekundärschädigung des Rückenmarks durch die Hypoxie zu minimieren, sollte eine optimale Oxygenierung ggf. durch frühzeitige Intubation angestrebt werden. Einer systemischen Hypotension ist durch ausreichende Flüssigkeitszufuhr entgegenzuwirken.

Die hoch dosierte Kortisongabe bei der akuten Querschnittlähmung stellt eine Therapieoption dar, wird jedoch nicht mehr generell empfohlen.

Die hoch dosierte Kortisontherapie in der Akutphase nach einer Querschnittlähmung ist nach wie vor Gegenstand der Diskussion. Im Rahmen des NASCIS-IIISchemas [1] wird zunächst ein Bolus von $30 \mathrm{mg}$ pro $\mathrm{kg}$ Körpergewicht Methylprednisolon verabreicht. Danach erfolgt eine weitere Gabe von 4,5 mg pro kg Körpergewicht pro Stunde über 23 Stunden.

Aufgrund methodischer Probleme der NASCIS-Studien wurden die Ergebnisse sowie die therapeutischen Konsequenzen in den letzten Jahren infrage gestellt. Außerdem wurde über eine Zunahme insbesondere schwerer pulmonaler Komplikationen nach hoch dosierter Kortisongabe berichtet. Die Amerikanische Gesellschaft für Neurochirurgie bezeichnete daher die hoch dosierte Prednisolongabe nach akuter Querschnittlähmung als „Option und nicht als Guideline“" [5]. Zum gleichen Ergebnis kam die Schweizer Konsensusgruppe um Jeanneret und Mäder. Auch hier wird festgehalten, dass Methylprednisolon in der o.g. Dosis in ausgewählten Fällen von spinalen Traumen weiter gegeben werden kann, jedoch eine generelle Empfehlung hierzu nicht gegeben werden kann [7].

Zahlreiche andere Substanzen wie GM1-Ganglioside, Naloxon und Nimodipine wurden zur Therapie der akuten traumatischen Rückenmarksläsion nach zum Teil vielversprechenden Ergebnissen im Tiermodell in verschiedenen Studien untersucht. Es konnten jedoch keine positiven Effekte auf das neurologische Outcome nachgewiesen werden [8]. 
Bei bestehender Blasenlähmung ist ein Blasenkatheterismus erforderlich. Idealerweise wird unter Berücksichtigung der Kontraindikationen ultraschallgesteuert eine suprapubische Ableitung angelegt, da hierdurch eine optimale Infektprophylaxe erreicht wird. Außerdem werden auf diese Weise zusätzliche Harnröhrenverletzungen vermieden, die einen später notwendigen regelmäßigen Einmalkatheterismus erschweren oder gar unmöglich machen [10].

\section{Operationsindikation}

Zeigt sich im Verlauf der Diagnostik eine Verletzung der Wirbelsäule als Ursache der traumatischen Querschnittlähmung, so wird diese in aller Regel operativ therapiert. Ziele der operativen Behandlung sind die rasche Dekompression des geschädigten Rückenmarks bei stellungsoder fragmentbedingter Kompression und die Stabilisierung der Wirbelsäulenverletzung, die eine rasche Mobilisation des Patienten ermöglicht und somit die Rehabilitationsfähigkeit herstellt.

Bei dislozierten Verletzungen der Halswirbelsäule ist eine geschlossene und möglichst exakte Reposition der Wirbelsäule die schnellste und wirksamste Dekompressionsmaßnahme [2]. Lässt sich eine stabile Reposition erreichen, kann je nach Allgemeinzustand des Patienten auch eine verzögerte Stabilisierung der Wirbelsäule zur Herstellung der Rehabilitationsfähigkeit sinnvoll sein. Der optimale Zeitpunkt der operativen Intervention wurde kontrovers diskutiert [9]. Einigkeit besteht darin, dass eine Zunahme des neurologischen Defizits während der präklinischen Phase oder bei der Reevaluation nach diagnostischen oder therapeutischen Maßnahmen eine absolute Notfallindikation darstellt. Bei inkompletten Lähmungen ASIA C und D wurden nach frühzeitiger operativer Dekompression und Stabilisierung gute Erholungsraten beschrieben, sodass in diesen Fällen eine dringliche Operationsindikation besteht $[4,6]$. Bei Kontusionen des Myeloms ohne Wirbelsäulenverletzung ergibt sich die Indikation aus dem Ausmaß der Spinalkanalstenose und dem zeitlichen Verlauf der Ausfallsymptomatik.

Akute Querschnittlähmungen nach Verletzungen der Brust- und Lendenwirbelsäule stellen eine dringliche Operationsindikation dar. In der Regel kommen als Notfallbehandlung bei der akuten Verletzung dorsale Stabilisierungsverfahren

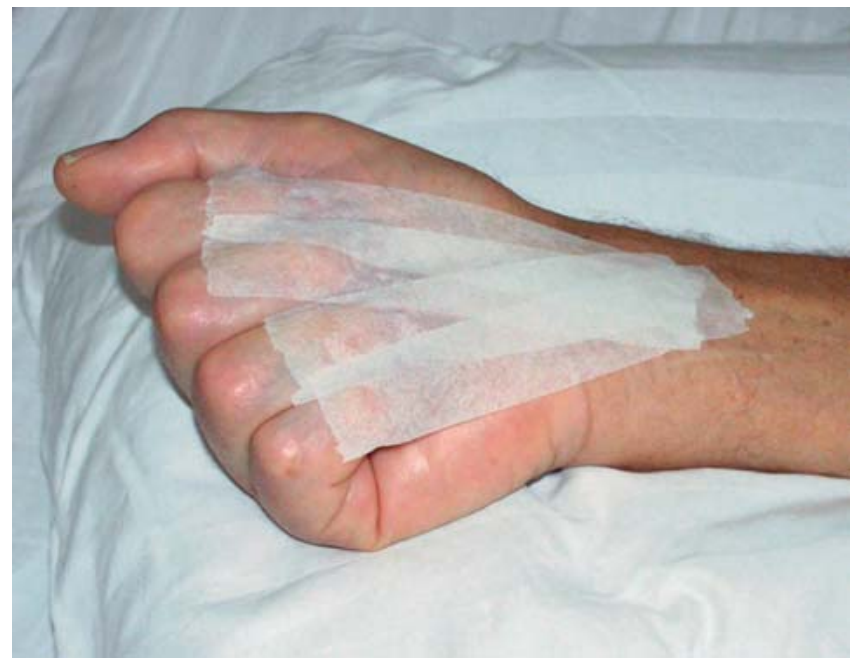

Abb. 2 Intermittierendes Taping der Hände eines tetraplegischen Patienten durch die Ergotherapie bereits auf der Intensivstation zur Vorbereitung einer „Funktionshand“.

zur Anwendung. Wenn erforderlich, kann in gleicher Sitzung eine dorsale Dekompression des Spinalkanals durchgeführt werden. In mehreren retrospektiven Studien konnte gezeigt werden, dass die frühzeitige operative Dekompression und Stabilisierung der Wirbelsäule zu einer Verbesserung des neurologischen Outcomes führt [4].

\section{Intensivtherapie}

Bereits auf der Intensivstation müssen bei akuter Querschnittlähmung je nach Lähmungsniveau und Lähmungsausmaß frührehabilitative Maßnahmen durchgeführt werden. Eine adäquate Lagerungsbehandlung zur Vermeidung von Druckstellen und Dekubitus ist hierbei obligat. Des Weiteren ist bereits in den ersten Tagen nach Eintritt der Lähmung eine physio- und ergotherapeutische Behandlung zur Vermeidung von Kontrakturen erforderlich. Bei Störungen der Handfunktion können hier auch spezielle Lagerungen (Abb.2) der Hände erforderlich werden. Zur Thromboseprophylaxe wird gemäß der Leitlinie der Gesellschaft für Neurologie niedermolekulares Heparin in an das Körpergewicht angepasster Dosierung empfohlen.

Eine regelmäßige Lagerungstherapie ist zur Verbesserung der Lungenfunktion und zur Vermeidung von Druckulzera zwingend notwendig. Bei tetraplegischen Patienten mit ausgeprägter respiratorischer Insuffizienz ist häufig zur Erleichterung des Weanings eine Tracheotomie erforderlich. Bei der Indikation hierzu sollte ebenso berücksichtigt werden, dass bei tetraplegischen Patienten sowie Patienten mit einer hoch thorakalen Paraplegie die Sekretmobilisation durch die reduzierte Kraft beim Abhusten längerfristig deutlich erschwert ist.

\section{Sekundäre Stabilisierung der Wirbelsäule}

Bei hochgradig instabilen Verletzungen der Halswirbelsäule kann es nach primär ventraler Spondylodese erforderlich werden, eine sekundäre dorsale Spondylodese durchzuführen, um eine im Rehabilitationsverlauf auftretende Schraubenlockerung mit entsprechender Dislokation zu vermeiden. Im Thorakolumbalbereich ist nach primär dorsaler Instrumentierung ggf. eine ventrale Spondylodese erforderlich. Diese sollte, wenn möglich, minimalinvasiv durchgeführt werden, um Sekundärschädigungen im Bereich der Thoraxwand und der Schultergürtelmuskulatur zu vermeiden. Das minimalinvasive Vorgehen hat hier aufgrund der geringen Morbidität bezüglich der weiteren Mobilisation des Unfallverletzten im Rollstuhl erhebliche Vorteile.

\section{Fortsetzung der Akutbehandlung im Zentrum}

Nach der Erstversorgung im Wirbelsäulenzentrum sollte so rasch wie möglich eine Verlegung in ein Querschnittzentrum zur Fortsetzung der Akutbehandlung angestrebt werden. In diesen Zentren erfolgt eine multimodale Weiterbehandlung unter Einbeziehung aller notwendigen Fachdisziplinen (Neurourologie, Neuroorthopädie, innere Medizin, Anästhesie und Schmerztherapie u.v.m.) und Berufsgruppen (Pflege, Physiotherapie, Ergotherapie, Psychologen, Sozialarbeiter, Sporttherapeuten u.v.m.). Ziel der Behandlung im Zentrum für Querschnittgelähmte ist es, dem akut 
verletzten oder erkrankten Patienten ein Maximum an Selbstständigkeit zu ermöglichen. Diese spezielle Möglichkeit der optimalen Primärversorgung in Spezialzentren und die Sonderstellung dieser Behandlung im DRG-System sollte im Interesse der schwer verletzten Patienten unbedingt erhalten werden. Nur so kann für den Patienten trotz der Querschnittlähmung, die seine Lebensbedingungen grundlegend verändert, sowohl eine medizinisch optimale Therapie als auch eine bestmögliche Wiedereingliederung in sein soziales Umfeld erreicht werden.

\section{Literatur}

${ }^{1}$ Bracken MB, Shepard MJ, Holford TR et al. Administration of methylprednisolone for 24 and 48 hours or tirilazad mesylate for 48 hours in the treatment of acute spinal cord injury. Results of the Third National Acute Spinal Cord Injury Randomized Controlled Trial. National Acute Spinal Cord Injury Study. JAMA 1997; 277: 1597-1604

2 Brunette DD, Rockswold GL. Neurologic recovery following rapid spinal realignment for complete cervical spinal cord injury. J Trauma 1987; 27: 445-447

${ }^{3}$ Exner G. Der Arbeitskreis „Querschnittlähmungen" des Hauptverbandes der gewerblichen Berufsgenossenschaften in Deutschland. Trauma Berufskrankh 2004; 6: 147-151

${ }^{4}$ Gaebler C, Maier R, Kutscha-Lissberg $F$ et al. Results of spinal cord decompression and thoracolumbar pedicle stabilization in relation to the time of operation. Spinal Cord 1999; 37: 33-39

${ }^{5}$ Hardley MN. Pharmacological therapy after acute cervical spinal cord injury. Neurosurgery 2002; 50 (Suppl. 3): S63-S72

${ }^{6}$ Hofmeister M, Bühren V. Therapiekonzept für Verletzungen der unteren HWS. Orthopäde 1999; 28: 401-413

7 Jeanneret B, Mäder M. Hochdosiertes Methylprednisolon in der Behandlung des akuten
Querschnittverletzten. Schweiz med Forum 2008; 8: 258-259

${ }^{8}$ Kwon BK et al. Strategies to promote neural repair and regeneration after spinal cord injury. Spine 2005; 30 (Suppl. 17): S3-S13

9 Northrup BE. Timing of surgery in patients with spinal cord injury. In: Garfin SR, Northrup $B E$, eds. Surgery for spinal cord injuries. New York: Raven Press; 1993: 5-7

10 Schurch B, Dietz V. Akutversorgung und Therapie des Rückenmarktraumas. In: Wallesch CW, Unterberg A, Dietz V, Hrsg. Neurotraumatologie. Stuttgart: Thieme; 2005: 165-172

\section{Dr. med Andreas Badke} Leitender Arzt Sektion Wirbelsäulenchirurgie

BG-Unfallklinik Tübingen Schnarrenbergstraße 95 72076 Tübingen

abadke@bgu-tuebingen.de 